\title{
Frontiers of shopper marketing, customer engagement and nonlinear creative - An interview with Peter Ingram, founder of BlendedBusiness
}

\begin{abstract}
Peter Ingram
brings The Fifth Channel over 20 years of integrated marketing experience, having counseled numerous Fortune 500 clients and national marketing agencies on national marketing strategy. In addition to his role with The Fifth Channel, Ingram is also the founder of BlendedBusiness, a consultancy focused on retail and shopper marketing strategy and implementation. Ingram was the Chief Marketing Officer of a major marketing automation company before launching The Fifth Channel and

BlendedBusiness. He has started divisions of major agencies including Fahlgren Advertising and Grizzard Communications, the latter a subsidiary of Omnicom Group, the world's largest holding company of marketing agencies. He was a founding partner in several marketing-related high-tech startups and continues to advise a number of these companies today. He is a recognized thought leader in shopper and retail marketing.
\end{abstract}

\begin{abstract}
Michael Moon interviews Peter Ingram, Founder of BlendedBusiness, on marketing integration and automation within a marketing operations platform. This interview features a discussion on customer relationship management and how marketers and their agencies are looking for new ways to optimize customer engagement. In addition, Moon and Ingram review current challenges in supply chain management as well as the change management required within organizations to better orient themselves around retail marketing and shopper marketing.
\end{abstract}

Journal of Digital Asset Management (2010) 6, 97-108. doi:10.1057/dam.2010.10

Keywords: shopper marketing; retail marketing; marketing automation; marketing integration; promotions; digital out-of-home

MM: Peter, start off with a little bit of your personal background and career highlights. PI: Sure, I am Peter Ingram, Founder of BlendedBusiness, which has been around for roughly 4 years. BlendedBusiness was formed to help clients who are either retailers, brands or marketing service providers serving retailers or brands, helping these stakeholders to better understand the impact of shopper and retail marketing on their business and helping to move them in the best direction.
Quick background on me, I have been out

in the marketing world for over 21 years.

Much of my career has been focused on agonies that deal with retailers or brands. I have led the marketing and sales for a couple of companies who are in the marketing automation space.

MM: And those companies include?

PI: Companies including an Omnicom agency called Grizzard Communications, where we implemented a turnaround effort for a print division, converting ultimately the business model to print on demand and fulfillment, ultimately using web-based tools that would drive highly customizable content out to the field. That is where I began my collaboration with my current partner, Carlos Gaviria. Other companies included Synq Solutions, which is another leading marketing automation company heavily based in the print side and fulfillment side of the business. Also in the early stage of 
my career, I helped McCann Erickson launch a division of their event marketing company in Atlanta. Ultimately, that morphed into what is now known as Momentum, and I started an Event Promotions Division for a Regional Agency, Fahlgren. Throughout my career, the common factor has been serving clients who are widely distributed types of organizations with multiple locations, a large number of consumers geographically dispersed, as well as employees and business partners who are geographically dispersed. Common issues have always been getting content to their recipients that is relevant and fairly optimized to the end target. Of course as time has gone along, technology has become a bigger and bigger part of the picture in how to solve those issues.

MM: Correct. And could you give us a little bit of a background or summary of BlendedBusiness in terms of your clientele and areas of practice? PI: Sure, BlendedBusiness was formed 4 years ago. I had left the Chief Marketing Officer post at Synq Solutions and begun to work predominantly with marketing service providers. Most of my time was spent serving in virtual executive posts for my clients. One of those was a leading Point-of-Purchase (POP) company. I helped this company to evolve its positioning from being a printer at the core really stuck at the bottom of the marketing services food chain - to repositioning itself as more of a strategy and thought leader for its clients, the likes of whom are companies such as Shell, ConocoPhillips, Valero, many in the convenience of gas category essentially, and quick-service restaurants such as Popeye's and Whataburger and Krystal. I helped this company that sought to delve into some new media types that were relevant in terms of the marketing environment for some of these clients that I have mentioned, and this newest venture for them was digital signage. Also, I worked with The Home Depot on some very belowthe-radar work to help them bring optimization to their signage management system. I helped a mobile marketing company go to market in what is a very cluttered marketplace, and along the way I also provided counsel to a variety of the clients who were served by these companies, including some of those I have mentioned.

MM: It seems to me, Peter, that in one shape, form or another you have had a career in which you maintained a primary focus of orchestrating the various activities and service providers of what we might call the 'marketing supply chain'. I use the term marketing supply chain to describe ultimately a network of individuals and businesses that support the marketing and sales processes of B2B or B2C companies. PI: Well, kind of extending off of what you were describing here, which pretty much aligns with the thinking that I have had and with the way in which I really needed to work to help clients master the supply chain a little bit better. I would describe it as a variety of media-types, a variety of content creation. Basically, stakeholders who are in the process of creating content and assets for marketers and those who provide end points to which those elements are deployed. So we will be talking about those who are, for example, agencies who would feed certain bits of content and strategy into the supply chain. We would also be talking about end-vendors who are responsible for taking elements of that content and strategy and applying it to a particular medium. Those could be print elements, POP, print advertising, outdoor billboards. All of those are either at the front or back end of the supply chain, creating or presenting the content in question, but then there are those sitting in the middle, like my company, who help to aggregate all of the information that was being produced and then distribute it back out to the end points that were relevant.

MM: Peter, this calls attention to two things that I would like you to develop. First, explain the orchestration function of a marketing operations platform, and how orchestration has begun to alter the configuration of business entities of marketing supply chains. Second, address the emerging, if not critical, role of a bidirectional flow of information up and down the supply chain, ultimately contributing to a feedback loop on the efficiency and effectiveness of the overall marketing spend. Speak to one or either of the points of orchestration in a marketing supply chain, or the emergence of marketing feedback systems? PI: In terms of coordination, one of the big challenges out there today for many agencies is that much of this is shifting to a very data- and digital-oriented nature. Of course, ultimately so much emphasis is being placed on hyper-local 
marketing and how one deploys content to any given end-point and to what that content is doing in terms of performance. Coordination now includes the setting all of those metrics and the tying together of these disparate media.

MM: In fact, we could even go further to say that most marketing organizations and the firms that serve them, find themselves undergoing an Information and Communication Technologies transformation of their operations.

Many marketing organizations must address the basic processes of how they create content and services and bring them to market; how these processes are wholly or in significant part digital, and as a function of that, require a digital infrastructure for managing these digital processes. PI: Absolutely, absolutely. I think one of the struggles that we are seeing out there in the agency community; you know, especially if you look at the lead agency structure for large marketers, the dynamics change in terms of what the areas of expertise need to be. For example, the simple flow of work has changed. There is now a whole new methodology for how content is created, for how strategy for this is created in the first place. Now that we are looking at this bidirectional flow of information and a bidirectional one-to-one dynamic relationship with individual targets or consumers, that changes a whole series of things. I think, we have all been seeing what the agencies are grappling with and what that means to them. It is changing things on a number of levels ranging from compensation models pay for performance, for example - changing the types of tools that are used and providing it to clients to assess, to create, and then to monitor and assess and adjust campaigns (dashboards are increasingly kind of a standard here), but the change is also from mass media to very much of a one-to-one type of focus. That sort of practice of segment of one essentially now needs to be applied to just about every medium out there. For those who are generalists, this is a tough shift. Conversely, for those who have been sitting in the direct and digital marketing space, they are well-positioned in many ways to take their best practices and place them in the lead. We are seeing some trends now where digital shops are being placed into the lead agency role. That is a huge sign of the shift here, huge sign.
MM: So, underlying this shift from traditional print and broadcast agencies to more of the digital interactive and/or direct agencies we find the emergence of multichannel analytics, and systems for listening to the voice of the customer - with the goal of optimizing levels of customer engagement and long-term value of customer relationships.

PI: Yes, an integrated and compliant way in multiple channels. Absolutely. Now there is a major shift, with the emphasis on Return on Marketing Investment (ROMI) for anything that is being done from the marketing and sales perspective these days. I just sat with a major brand within the last month that is undergoing an entire shift to how they budget. It is kind of a fluid (I think as they called it fluid) budgeting process, meaning at

the core, everything within their marketing must perform and be measured against some sales increase, so it is no longer enough to measure brand awareness and have that be the end of the day. Everything with this particular company must tie back to sales, which is a whole change management proposition for the organization, one where everything now must tie back to essentially a scorecard that is measured at the highest level based on sales performance.

MM: Peter, this reflects a deeper transformation of business, a kind of maturation of the balance scorecard. I'm thinking, specifically of a set of metrics for measuring the performance of intangible assets, emphasizing more than ever the performance of the brand and customer value propositions. I believe that the balanced scorecard offers a framework for measuring return on marketing investments beyond the immediate marketing spend, using more evidence- or data-based facts for measuring the effect of marketing spend.

PI: Absolutely, absolutely. Everything must be measured for effectiveness and things that are underperforming will be dropped. And as I was referring to this brand discussion, that space, you know, goes, so for example, their model will be very different. They will not establish budgets upfront for the year; they are not going to commit to all media types. As a result, it is very organic as it flows. It will mean a whole new way of managing campaigns for this client and that kind of goes back to this marketing 
supply chain orchestration issue, because it is getting increasingly complex as we talk about things such as the various customer personas and the various scenarios that we have attached. MM: Well, Peter, as marketing becomes much more event-driven and contingent, management of the marketing process entails more than what we have called 'marketing procedural' traditional old-school marketing that starts off with some customer or consumer insight that leads into some sort of a strategic planning process to determine if that is a market segment. If that segment opportunity looks worthwhile, the planning process produces a marketing strategy comprising one or two big insights, a core theme and a set message. This forms the basis of a marketing plan, detailing targeted routes to market, key results, media mix, and key messages by segment, and so on.

The marketing plan supports the development of a set of creative briefs, often a specific set of communication and messaging goals by channel or medium, including packaging, in-store merchandizing, direct mail, online, mobile, guerrilla, street teams, whatever.

Each creative brief kicks off a set of parallel, concurrent workflows; each with their own review and approval process and creative development, ideation, refinement, approval, production, mastering and distribution.

Some weeks later, out pops the content ready to go to market, converging on a calendared date and the execution of the marketing campaign with advertising, trade marketing, outdoor, direct mail, telemarketing, in-store merchandizing, etc. - all hit hopefully at the time, creating an echo or a synergistic effect in the market. Months after the strategy meeting, we start to see what if any from sales resulted.

And that procedure is anywhere from 3 to 6 months in length and kind of what you are saying is that the major shift underway is the marketing procedural while still will remain viable for point of the foreseeable future, specifically around product launches and stuff. We are seeing the emergence of something that is far more contingent, far more opportunistic, and then something that happens in the matter of days or weeks as opposed to months and that really kind of throws a wrench into the whole traditional budgeting and planning process as well as the execution process, where we now have to execute in days or weeks or in most cases hours and our execution is no longer, you know, the model you described as very much the old school sort of push model in terms of content and simply camping deployment, but as you know, we would talk about now, you know, I suppose a way to describe this would be that I imagine that as we get down the path with this new marketing procedural sort of approach that you are describing much more automated, triggerbased, real-time, analytics-driven. One of the things that I envision marketers doing is still what, you know, the planning of promotions let us say where perhaps you have a promotion every month or every quarter. You may still as a marketer sort of create the dramatic template for that.

PI: Right, so there is a sponsorship, there is a looking field, there are things such as this, but the campaign is ultimately in many ways triggered by the consumer. You could almost imagine that the consumer is part of that creative team, so as I have needs and wants and very much dependent upon my location, the time, the medium that I am dealing with, I kind of create the final version of that campaign on the fly myself and that may be done through triggers that are not consciously flipped by me. It may be that I simply purchased a product, but in another instance, I may raise my hand and indicate an interest in a certain thing now, so rather than the brand pushing all the stuff to me in the hope that something sticks, it is a bit of reverse of that and that does throw a whole wrench into the works for marketers, because how I do deal with that, how do I listen to my consumer when they are talking to me, how do I know where they are, how do I ensure that what I provide to them is not only relevant, but is entirely as we would say, entirely actionable meaning. If I am standing in a store with a mobile device and I have by virtue of being a member of a Loyalty Program, and a coupon has drawn me into the store, a real-time issue coupon, well, that is great, but I better be able to find that product in the store, I better be able to get the discount, in other words the coupon should be absolutely redeemable. If anything less than that happens, I am now not happy, and there are other issues the brands 
now need to contend with, like social media. When the consumer is unhappy that virally can ramp up to a big issue right away, so that we have, you know this need now.

MM: Peter, one of the things that I want to come back to what you just mentioned is this notion of coupons and loyalty programs. Now, in the traditional mindset, marketers distributed coupons with free-standing inserts in daily and Sunday newspapers, clip-out ads in newspapers and regional newsweeklies, direct-mail value packs, and traditional direct mailer. Basically, all the ways to put coupons into the hands of budget-conscious consumers who would then clip, carry and redeem them at points of sale; which would include barcode that would get scanned in along with the purchase right?

PI: Right.

MM: And the whole idea of coupons is undergoing a digital transformation. Increasingly, marketers distribute coupons over the web, where I download and print them, where I can get coupons from aggregators who basically publish online sortable collections of coupons by brands or category as well as mobile coupons that I can redeem as a function of bluetooth or some sort of wireless or optical connection.

Now with the expansion of Facebook, Twitter, Loopt and other Web 2.0 firms, we have come to see geo-tagged social coupons; specifically, geo-tagging of coupons uses the geo-location function of mobile phones as well as their 'presencing capabilities' (akin to your 'I'm online' or 'I'm busy' status in instant messaging or Skype).

I have even seen the next generation mobile coupons; as the coupon bearer enters the shopping mall or comes through the retailer's door, the location-aware coupons signal back to the store that a coupon holder $X$ just showed up. Smart retailers might then want to take an appropriate action, or, the smart mobile coupon might even trigger a particular point of sale display, activating a particular message as it relates to that particular personnel consumer. PI: Absolutely.

MM: Another thing that we have seen in the coupon area entails social tagging of coupons. For example, I might go to Facebook or a specialized social network where I find and download a coupon, not necessarily for me, but for those friends who are part of my personal crew or professional social network. So then I pass this coupon off to you, the coupon records the transfer as well as builds my account of social points or social scrip - redeemable for cash money, discounts or special access privileges.

PI: Right.

MM: Thus, social coupons maintain a chain of evidence in terms of who downloaded, transferred to whom and redeemed. This gives rise to a whole new dimension in multilevel marketing and what are the implications of all that?

PI: And if you are asking me that question, I would think one of the implications is that it is a whole heck of a lot for the marketers to grapple with. I think the key thing is as you described here - it's all about building your databases to be able to accommodate this information. Not only to simply account for it, but also to be able to adapt to changes in those variables on the fly, because of course that will then trigger some sort of a communication, a bi-directional communication with some individual or venue or item dynamically, real time. That's a complicated media model, to say the least. I should also say that one of the things that I am seeing out here right now is that there is a lot of talk about social, there is a lot of talk about mobile, certainly as it goes with mobile and retail, there is lot of discussion about mobile couponing, digital couponing in general. As it goes with some of these things, mobile couponing is a good example. Lots of discussion about it, but frankly as it goes today, the typical consumer does not own a smart phone, so there has been a lot of discussion about smart phone applications and iPhone, but what I have been seeing is a lot of one-off treatments of new media applications without necessarily a lot of thought about how to tie this back into a platform that also suits the masses. If I am a mass marker and my intent is to reach the masses, then a discussion around an iPhone application is not as relevant necessarily as perhaps the discussion around how I could do something with text messaging, something that would reach the average mobile device user, and be effective while mapping out for the future. So, I see a lot of confusion in the marketers. There is interest in testing and piloting, but there has not been a lot of 
commitment to long term with the new media stuff yet, understandably lots of answers yet to be had, but I see that there are some really fantastic end points out there, but they are not mature yet. Digital signage would be another example of that. You used the example of a consumer walking into, say, a mall and my GPS (which by the way is not very useful once you walk inside the confines of a building), alerts somebody that I have walked into the building. Well, now I walk into the mall and my mobile device magically communicates with some sort of technology to alert that store that I am in their vicinity and which then pushes content to a digital sign that is sitting in the window that pulls me into the store. Maybe I also receive a coupon on my screen at the same time. All these things are coming, but also there is a challenge in terms of the marketing supply chain. We have to remember the consumer, the end-user and end-target here. For the consumer, let's use that as an example: a consumer walks into the mall. If I have an iPhone that is GPS-enabled, but do I have the application open that would enable the communication to flow back and forth between my device and that of the retailer that we talked about. I have to know to turn on that application, and then if I am going to communicate with Barnes and Noble, the first retailer encounter through Wi-Fi, I must turn on Wi-Fi on my phone. If I am going to communicate with another retailer, I might need to have my bluetooth radio enabled. These are things that the consumer till today has to do; there has to be a conscious action by the consumer.

MM: So clearly, Peter, the future arrives unevenly distributed and some futures will arrive later than others, and certainly this is true of shopper marketing. Perhaps you can give us a kind of what-is-going-on update in shopper marketing.

PI: Sure, and of course some of the media types are end points we have talked about here that affect shopper marketing and, by the way, depending upon who you talk to, the definition of shopper marketing will change. Traditionally, many talk about shopper marketing and really are thinking about communicating with consumers while they are in store.

MM: Sometimes called in-store marketing right?
PI: In-store marketing right or on premise. However, in large part thanks to some of these new media types we talked about here mobile, digital, web, social media - the argument that we would make is that the consumer could be considered a shopper when they are not in the store or a consumer could be considered a shopper for retailer $B$ while they are standing in retailer $A$, so the line starts blur. As it does, what it does mean, of course, is really now for consumers to be fulfilled or for the promise to consumers to be fulfilled by retailers and brands, there needs to be a much more dynamic relationship established that is very real time in nature. If you were to look at the experience one has at, say, Amazon.com, very personalized, everything is right at your fingertips, optimized for you. That is where it needs to head or that is much of the discussion now for in-store.

MM: So, if I understand that right, you see an emerging discipline called 'customer experience management' as kind of the superset of user experience, user design for online system.

So, customer experience management entails the same mindset of user interaction, user design and information architecture within the context of customer experience management; only now customer experience management has made its way into the retail footprint of shoppers.

PI: Absolutely. Yeah.

MM: So one of the things that is inherent to customer experience management is the notion of a user persona or a customer persona that describes among other things, basic demographic and psychographic traits that ultimately lead to what we call engagement preferences, and therefore what message and/or what configuration of products should lead most logically present to this particular individual displaying this persona.

PI: Yes, right, so taking into account the scenario, yeah.

MM: And so as we take that notion of customer experience management into the retail store, how does that translate? What happens? What are some of the innovations at retail that will affect customer experience management? PI: Well, you are seeing a variety of technologies introduced, I think one of the challenges I should throw out as the caveat here is that lots of technologies are being 
introduced; however, the big challenge that has not yet been tackled well is the integration of the operations supply chain with the marketing supply chain. But, that being said, some examples of the kind of technologies that are hitting retail that are intended to address the customer personas, which provides a mechanism for managing scenarios that are between the consumer and the retailer and the brands, things such as stuff we have seen in the store for sometime in one form of fashion. For example, interactive kiosks: those are getting smarter, they are usually tied into loyalty marketing programs. The real underpinning of all of this stuff is going increasingly toward loyalty marketing platforms and how to activate the consumer in store. Ultimately, the loyalty platform is the optimal way to get the consumer to engage. It is in the world of retail that the brands and retailers tend to agree that as it goes with shopper marketing and retail marketing, the retailers really carry the torch there and, not surprisingly, they are the funnel to drive the consumer to a transaction in the physical world, but other technologies we are seeing that hit that space include things such as what we have talked about: digital signage, mobile marketing technology, self-checkouts. This kind of ties into the kiosk arena. We are now seeing that even retailers are starting to deploy Wi-Fi networks and things like that, which are intended to provide better customer experience if only for the convenience of being able to use Wireless Application Protocol, say on your mobile device while walking the aisles of a store. But, the intent is or the challenge right now is that many of these technologies are being explored and as you have said earlier, a very special fashion, very one-off type of applications. I'm speaking in generalities, but the problem has been that some of these solutions really are set off and one-off tests, they are not very tightly integrated, often times even with loyalty systems themselves. Even though there may be a one-to-one exchange that is being created between the retailer and that consumer, there may not be a direct connect with the loyalty database, so it's tough to measure these media types effectively when they are not really tied into the traditional operating model of the marketer. It is coming, but those are some of the big, big challenges and kind of get back to what you were talking about earlier, tagging assets that go all the way down, better data with better tagging of consumer profiles, better tagging of the assets used to communicate with the consumers and a better integration of all the tools that would be used to drive communications to those consumers.

MM: Let's examine another dimension of the shopper marketing phenomena. First, shopper marketing entails collecting a lot more data and using that data in a lot of different analytic modes. And to that end, companies like Teradata, SAS, Oracle and IBM now offer very deep, rich and broad analytic disciplines around how to slice and dice not just loyalty program data, but melding that in with other sources of data, say for example web site data, syndicated data and so on.

PI: Yeah.

MM: Second, shopper marketing needs enriched data from sources such as Epsilon, Acxiom or Experian - consumer database compilers. Third, shopper marketing requires a higher level of cooperation and trust among packaged goods brands and retailers.

Clearly, the retail organization owns the customer relationship. Their customers come in on a regular basis, they got them in a Loyalty Program, they've got these consumers' transactional data, and the brand generally does not have access to a lot of that data.

The brand gets the consumer through a syndicated source like Information Resources, Inc. (IRI). Tell us how shopper marketing affects the relationship between brands and retailers. PI: Well, I guess what I would say is that to do shopper marketing well or in a holistic sense is treating the consumer by giving them that terrific experience where they get the information they need when they want it in terms that they expect, that it requires that brands and retailers collaborate better. That is not to say that the brands and retailers do collaborate more effectively or even willingly right now, but there is a lot of discussion out there right now about this. There are some things that you will see out there, initiatives that are underway. The In-Store Marketing Institute, for example, is midstream with something called the Retail Commission on Shopper Marketing, which is all about that. It is a group of key 
retailers and brands who are grappling with this very thing. What are some of these practices or how can we collaborate better? What are the ways in which we need to communicate with the consumer, and what instances might that be a shared customer between the retailer and the brand?

MM: In my conversations with people like you and others play active roles in this larger industry initiative of shopper marketing, I see three things that seem to have really stood out.

One, the overall marketing process has become much more data-driven, so much so that the analytic shops or capabilities required to manage this analytically driven marketing process has gone beyond the cognitive or skill sets of many marketers.

Two, the markets have become much more dynamic and contingent and nonlinear and as a function of that managing the marketing process begins to look more and more like real-time process control or an air traffic control system. This suggests that we need a whole team of specialists, all looking at data from different points of view and inferring certain insights from that. We might call those, engagement managers.

Third, the consumer really is responding to more of these kinds of open end engagements, conversations as opposed to these kind of more broadcast one-way intrusive insertions of brand messaging and are really wanting to having more fun, more interaction, more collaboration in the conversation around what is a brand and how does it contribute to me and my friends. PI: Yeah.

MM: Does this not call attention to the need for what we might call a "customer engagement agency?" Kind of the next generation agency that brings together the disciplines of Customer Relationship Management and business intelligence, web analytics and social media analytics, tightly coupled to creative? Not just tightly linked creative, content, but services, as well - interactive services - and then part of that process and agency responsibility becomes the facilitating of the delivery or provisioning of contents and services, respectively, to various brand touch points, including in-store? PI: Yeah, you know, I guess what I would say is, if I were a brand that is the sort of agency that, I would want in the lead, and frankly there really is not such an agency that I can think of today, not many, there are some who dabble in it well, but most of them tend to still live in their very siloed areas of specialty. I think that again the digital shops have a really good opportunity to step into the fore here, anyone who has been dealing with the data side of the business or position, but again they fall short in some of the areas where others are stronger, you know, promotions agency or an in-store agency has perhaps arguably a better understanding of the shopper in the motor world certainly, but that is the trend that is coming and I think that you are absolutely right, the clients are struggling. In general, they do not have the resources for all of this and these are a lot of new moving bits and pieces that were not dealt with at this level of granular level before. The granular stuff was not thought to be perhaps as valuable, but that is not what the consumer is telling everybody. I think we would all agree that the power is shifting to the consumer, so the clients are struggling with that: well, I do not have the resources to do this, I am looking for an agency to help me and by the way, as it goes, new channels seem to pop up every day that we need to take into consideration, and I do not know how to handle all that. That is an awful lot of manual work, to your point, lots of manpower that could be perceived to be placed against this kind of thing. That is where I think the technology side of things really comes into play here, you know: intelligent dashboards, business-rules-based systems that can facilitate a lot of this dynamic communication through multiple end points, dealing with multiple personas and scenarios, all at the same time and redeploying content and optimizing it to the individual and the scenario and the end point. All this has to flow seamlessly. As it goes with the consumer from a look and feel perspective, branding, messaging and so forth, what they see on their mobile device from brand $A$ needs to be entirely consistent with what they see on that billboard as they drive down that street or that static cling in the window of the store or that digital sign and the checkout counter. The compliance has to be there, so that it gets into a whole complex area that we think is best dealt with through technology. 
MM: You know, Peter, as we move toward the conclusion of our interview here, it seems to me that we are really talking about a key challenge for many marketing organizations. The challenge really seems to come down to how quickly and how effectively we can manage change. Companies that will do well in this nonlinear real-time contingent market space of ours will be those organizations that have developed strong operational capabilities for innovation and managing change. Can you speak of some of the things that you found that either works or perhaps does not work in that area of change management and innovation?

PI: Well, this gets a little beyond what we do as well, but using our change management is at the core of a lot, there is obviously, there is just list of all this stuff, and one of the big challenges is that especially in this particular economy, because this is all new and because everything is so highly measurable now and everything is essentially being placed into some sort of digital business rule environment. Meaning, in other words, I could map what anybody does. Your job description and function is now a business rule. That scares people because they are highly exposed, and particularly today, I think there is a perception that if we were to orchestrate change management through technology-oriented optimization that creates a lot of fear in people right now. There is a lack of trust that any individual has, so it is tough to get the change happening. But, it has to happen in a series of small steps. You know, a lot of what we are talking about here is the Holy Grail approach to dealing with the consumer, various personas and scenarios and dealing with them right now. This is a wonderful Holy Grail approach, but to move a massive organization toward that complete seamless treatment of the consumer, no matter where they are and when they are small steps, I think that the whole effort needs to start with things like you have mentioned, the balance scorecard, helping a client map out what they do, where we are headed, how to change and implementing through small things. MM: Well, the balance scorecard is simply going to give you a set of pretty good metrics, things for measuring the performance of intangible assets. Generally that leads to the place, 'Okay, now that we have got a feedback loop that induces us to a refine our overall corporate strategy, we need a better, more effective way to communicating our strategy throughout the firm'. And, that generally leads to them into using strategy maps to visually depict and explain a more salient, comprehensive strategy to everyone in their firm.

PI: I was going to say that brings to mind a white paper of yours that was issued not long ago. I am failing to recall the company that was featured, but there was a whole discussion around the deployment of strategy maps and the taking of the organization through the change management process.

MM: Now, the execution of the strategy requires a structured repeatable process for managing change. Yet, most companies that I have observed do not really have well-developed changed management processes. Many companies have good project management and change facilitation processes from IT, but lack even the basics for how to innovate new processes or innovate new services.

PI: And, I think you are right that one of the issues is that change management happens in an unplanned way in many organizations. But, the challenge too is that we are shifting to an IT- and data-oriented digital environment, and it brings to the fore part of the organization that was often times sort of set aside and sort of kept in the background - IT - the dynamic (and I am speaking probably a few good years back), between an IT organization and marketing or sales, especially marketing in my work, the dynamic there was not often that strong.

MM: Just in interviews that I conducted yesterday and the day before marketing in IT traditionally have an adversarial relationship and you know both of them come from completely two different tribes and they do not really understand how to play nice.

PI: That is right, and for those who are struggling with that, it is often simply a sort of a language and cultural barrier. But, the new marketing culture is necessarily inclusive of IT and data.

MM: And vice versa.

PI: That was not there before and vice versa. I really buy into what you say about the 
need to implement this change and to have a structure for that and the balanced scorecard and strategy map. It could be a great way not only to align all these disparate players, but also to make everything that we have been talking about happen. You know, the simple example that you mentioned of collaboration between retailers and brands, well that, that is a change management proposition within the retail industry just on its own. How do I convince these often times opposed stakeholders to collaborate better, when there's a love-hate relationship between the retailer and brand? Do I trust you to do well, to do the right thing at this date and not come around and turn around and use it to my disadvantage? That gets beyond the pure science of things, so we could engineer systems that enable that data flow back and forth and you know customize use of it, but unless we align the stakeholders well and get an agreement as to what the best practice will be that we will all adhere to, you know the rest just kind of struggles to happen. So, that gets back to what you are describing here. Change management working with a structure like the balanced scorecard and strategy maps are a great way to align for folks to a new way, but it requires somebody who has the expertise to help. Often times a third party, rather than somebody who is tied into the politics of the organizations at hand.

MM: Yeah, and that trust that the third party inevitably becomes a trusted intermediary between that brand and retailer, and I guess the case that I am making is that the trusted third party might be the next generation agency that is compensated perhaps both by the brand and retailer.

PI: Yeah, you know it depends on the initiative here. If I were looking at a general agency of the future for a brand, I am not sure that I could envision them being those change management agents as well. I think they would be partners to an organization that would help the client to optimize its structure and process. But, I do not think that I would see the agency taking the entire change management role on themselves. I think that they will be a part of that key group. I would see them as being a contributor to the design of the process and they would be the ones held accountable for implementing the process that is designed.
MM: So it falls into the old story line of 'Go get me a stick to beat you with'.

PI: Well, I think there is a lot of beating going on right now, out there.

MM: But the case that you make is that the agency is either a function of temperament and/or a business model that may not have the appropriate mindset position to really engage the brand and retailer as this trusted intermediary. So if not the agency then who? Does this call attention to traditional management consultancies?

PI: Well, in the traditional sense, I guess, that is where I would go, whether that is the entity or whether it is smaller firms, you know the TopRights of the world and so forth, the real boutique shops, the North Highlands, that is, I guess what I would have to confess where I go back to my traditional way of thinking about who the specialist in the space is. That is kind of where I start to think about first and that may simply be because this is a group of firms who have provided that service. If it were to be the agencies, I guess the thing would be if I were to turn to the agencies and I would task them with this, you know that there is a whole new competency that has to be introduced in the agency model and a whole new skill set that has to be brought in, maybe then you will start to see the Omnicoms of the world really pilfering consultancies for their talent.

MM: We may find another dark horse in this whole race of customer engagement. I submit that many of the large incumbent software companies in business intelligence will go to the brands and say, 'Hey, you really need a large trusted third party to broker the information and power with larger retailers. We are the only economic actors in the market who can really kind of wade into this contest with sufficient economic power to harmonize these traditional adversarial relationships between brands and consumers. Besides our rationale for doing that is that we have to make the market for our technologies and services. Technologies and services that all, by the way, often outstrip the resource base or the skill set of many of our natural buyers; so rather than wait around for our buyers to develop the operational capabilities, let's go solve that problem and earn good revenues'. Does this make sense to you? 
PI: I would agree that those who are already facilitating with a data communication are certainly in a good position to facilitate broader higher level communication as well, since, all this does tie back to data, the exchange of data, and compiling of the right data and acting up on it, analyzing and acting upon it appropriately and speedily. Yes, I could see those companies certainly sitting in a strong position. I would add if there is a way, this sort of business is a new venture, a separate venture.

One, that is all around this issue of how to facilitate some of the things that we are talking about here. At BlendedBusiness, we are creating a separate company for that. It is very much oriented around the best practices and the data flow and all of that. We are calling that company The Fifth Channel. You will probably hear more of that new business going forward, because it is about getting some of the stuff off the ground that you and I have discussed. But that aside, I think that some of the things we will see out there that are happening may flow through those, the Epsilon, even the Merkle. We've seen Merkle out there with some stuff recently on marketing integration and optimization - I think it was AdAge that had a big spread sponsored by Merkle talking a lot about this sort of optimization. There is great opportunity for companies like these, and I think ultimately one of the things that we hope to see happen - and we hope to be a part of this - is the creation of some tools that are almost neutral portals, portals where you might see this type of collaboration occurring with better effect between example retailers and brands. Where if I am a beverage marketer, I may chose to place a copy of my data. Maybe it is not the original, but I will place a copy of my consumer information, consumer data and product data on this portal as would my key accounts. My key retailers would also place certain bits of information about consumers and product Stock Keeping Units (SKUs) and other things and planograms into the system. There may be some business rules that would allow for the brand to see certain views of the consumer that would show their product segment or category, and vice versa that the retailer might see certain views of the consumer who buy the particular beverage marketers' products. These are not necessarily complete views of that same consumer's profile, but key views that are shared - this is a key component in the collaboration site.

I think that those who are dealing with these data are in the right position to create this better, new model. Are they necessarily the change management experts? I do not see that today, but I do not see that as not being an opportunity, it is just not what they do today. So I buy into what you are saying - there are certainly some changes that need to happen to make them that player.

MM: Well, you know this sounds like a great place to perhaps conclude our discussion today. However, before we do that, do you have any final remarks with which you would like to conclude?

PI: I would say we have really hit on the key of it that data are behind everything and analysis of that data for use for deployment of that information in various ways and deployment of assets in conjunction with that data is the key to it all. It requires a whole new way of aggregating that information. Where we used to talk about only the right information needed and did not build warehouses to accommodate more information than that, it is kind of going a different direction now, so those that are able to deal with that sooner versus later certainly are ultimately going to have a better chance of building that deeper, longer-standing, more meaningful, more valuable relationship with the consumer. So everything we have talked about here requires change management. All of that is all in the name of crafting a tighter relationship with the consumer, so I think it's a really interesting time of shift. Fifteen years ago we talked about direct marketing often times in my world of promotions as a necessarily evil: we might throw out a postcard alongside with everything else that we really were excited about - it has come full circle and we are now looking at direct marketing and digital marketing best practices, as really the drivers of marketing, so lots of change; ultimately the good news is the technologies are there, we have a lot in terms of end points, in terms of systems to manage this best. The lacking bits are in the intermediary roles on systems, but those two will come, so it is an exciting time, and we are 
glad to be in the middle of it. Along with that, it's critical to keep in mind best practices from the likes of Kaplan and Norton, which I think are essential. There has just never been so much to deal with marketers as there is today. So getting it all organized is really the key to the start.
MM: Great place to conclude. Peter, thank you so much for the opportunity to spend time with you today and to explore these issues and I look forward to many more conversations to come. PI: Likewise, likewise and it is a pleasure to talk with you always, Michael.

MM: Fabulous. 